\title{
Food authentication: Techniques, trends \& emerging approaches
}

Georgios P. Danezis, Aristidis S. Tsagkaris, Federica Camin, Vladimir Brusic, Constantinos A. Georgiou

School of Medicine

\section{Abstract}

Food authentication is a rapidly growing field due to increasing public awareness concerning food quality and safety. This review presents critically the analytical techniques which are used for authenticity assessment, explaining how and why they give plausible solutions. Classification of different methodologies is based on authenticity indicators providing insight into future developments. Analytical breakthroughs and novel techniques that emerged recently are discussed, along with their applications on food authentication. We have discussed current limits and gaps, related to informatics needs for data analysis of large quantities. Reporting standards and reference database are elaborated indicating urgent needs for the progress of this field. A scientometric evaluation highlighted the research trends and emerging approaches of this evolving field. Popular analytical techniques are commented, while the potential of the field is depicted in the temporal evolution of the research output focusing on geographical distribution of research activity and preferred journals used for dissemination.

\section{Original language English}

Journal $\quad \underline{\text { TrAC - Trends in Analytical Chemistry }}$

State Accepted/In press - 2016

Danezis, G. P., Tsagkaris, A. S., Camin, F., Brusic, V., \& Georgiou, C. A. (2016). Food authentication: Techniques, trends \& emerging approaches. TrAC - Trends in Analytical Chemistry. DOI: 10.1016/j.trac.2016.02.026 